\title{
残余应力对 $\mathrm{SrRuO}_{3}$ 薄膜磁学及电输运性能的影响
}

\author{
朱明康 ${ }^{1,2}$, 董显林 ${ }^{2}$, 陈 莹 ${ }^{2}$, 丁国际 ${ }^{1}$, 王根水 ${ }^{2}$
}

(1. 上海大学 环境与化学工程学院, 上海 200436; 2. 中国科学院 上海硅酸盐研究所, 上海 200050)

摘 要: 采用射频磁控溅射法在单晶 $\mathrm{SrTiO}_{3}(\mathrm{STO})$ 衬底和硅 $(\mathrm{Si})$ 祄底上制备出不同取向的 $\mathrm{SrRuO}_{3}(\mathrm{SRO})$ 薄膜，对薄 膜的残余应力进行了分析, 并研究了应力对不同取向 SRO 薄膜磁学性能与电输运特性的影响。根据 X 射线衍射 $(\mathrm{XRD})$ 结果分析可知, $\mathrm{Si}$ 基 SRO 薄膜为多晶单轴取向薄膜, 且应力来源主要为热失配拉应力; STO 基 SRO 薄膜为外 延薄膜, 其应力主要为热失配压应力和外延压应力; 磁学性能测试表明, (001)取向 SRO 薄膜比(110)取向薄膜拥有 更高的居里温度 $T_{\mathrm{C}}$; 压应力提高了(001)取向 SRO 薄膜的 $T_{\mathrm{C}}$, 却降低了 $(110)$ 取向薄膜的 $T_{\mathrm{C}}$ 。电阻性能测试表明, 对 于在同种类型祄底上沉积的 SRO 薄膜, (001)取向的薄膜的剩余表面电阻比 $(R R R)$ 高于 $(110)$ 取向的薄膜。另外, 拉应 力引起了薄膜微结构的无序度增加，弱化了表面电阻率的温度依赖性，提高了金属绝缘体转变温度 $\left(T_{\mathrm{MI}}\right)$ 。

关 键 词: 钓酸锶; 取向; 残余应力; 磁学性能; 电输运特性

中图分类号: TQ174 文献标识码: A

\section{Effect of Residual Stress on Magnetic and Electrical Transport Properties in $\mathrm{SrRuO}_{3}$ Thin Films}

\begin{abstract}
ZHU Ming-Kang ${ }^{1,2}$, DONG Xian-Lin ${ }^{2}$, CHEN Yin ${ }^{2}$, DING Guo-Ji ${ }^{1}$, WANG Gen-Shui ${ }^{2}$
(1. Shanghai University, School of Environmental and Chemical Engineering, Shanghai 200436, China; 2. Shanghai Institute of Ceramics, Chinese Academy of Sciences, Shanghai 200050, China)

Abstract: A series of $\mathrm{SrRuO}_{3}$ (SRO) thin films with preferential orientations were grown on $\mathrm{SrTiO}_{3}$ (STO) and $\mathrm{Si}$ substrates respectively by radio frequency (RF) magnetron sputtering technique. XRD results show that STO-based SRO thin films are epitaxial which differ from the one-axis orineted Si-based films. Residual stress type of the deposited films and effect of the stress on magnetic and electrical transport properties were systematically analyzed and summarized. STO-based SRO films suffer from compressive stress due to the lattice and thermal mismatch, while the Si-based films are subjected to tensile stress which is only derived from the thermal mismatch. The compressive stress promotes the Curie temperature $\left(T_{\mathrm{C}}\right)$ of (001)-oriented SRO films, but reduces the $T_{\mathrm{C}}$ of (110)-oriented SRO films, which may be due to the different states of rotation and tilt of $\mathrm{RuO}_{6}$ octahedron. Besides, the (001)-oriented SRO films possess higher $T_{\mathrm{C}}$ than the (110)-oriented SRO films all along. The results of temperature versus resistivity measurements reveal that residual resistivity ratio (RRR) of (001)-oriented SRO films is higher than that of (110)-oriented SRO films which deposited on the same substrate. Moreover, the temperature of metal-insulator transition $\left(T_{\mathrm{MI}}\right)$ increases from $16 \mathrm{~K}$ to $32 \mathrm{~K}$ while the temperature dependence of resistivity is suppressed by the tensile stress.
\end{abstract}

Key words: $\mathrm{SrRuO}_{3}$; orientation; residual stress; magnetic properties; transport properties 
$\mathrm{SrRuO}_{3}(\mathrm{SRO})$ 是 $4 \mathrm{~d}$ 过渡金属氧化物中唯一既具有 铁磁性又具有金属导电特性的物质, 其结构为正交畸 变钻铁矿结构 $(a=0.557 \mathrm{~nm}, b=0.553 \mathrm{~nm}, c=0.784 \mathrm{~nm})$, 也可看作是轻微扭曲的噟立方结构 $(a=0.393 \mathrm{~nm})^{[1]}$ 。 室温下, SRO 表现为顺磁性, 但当温度低于 $160 \mathrm{~K}$, 则表现出巡游铁磁性 ${ }^{[2]}$ 。铁磁性的 SRO 垂直剩余磁 化强度大, 因而可在磁性隧道结和磁存储器件中得到 应用 ${ }^{[3]}$ 。此外, SRO 还具有较高的电导率、较好的化 学和热稳定性, 并且其钙铁矿结构与目前广泛研究的 铁电材料 $\left(\mathrm{Pb}(\mathrm{Zr}, \mathrm{Ti}) \mathrm{O}_{3}, \mathrm{BaTiO}_{3}\right)$ 晶格匹配良好 ${ }^{[4-6]}$ 。因此 SRO 成为铁电薄膜器件理想的电极材料, 而广泛应 用于铁电随机存储器(FeRAM)、微电子机械系统 (MEMS)以及非制冷红外焦平面阵列等器件中 ${ }^{[7-8]}$ 。

针对 SRO 薄膜的研究已开展多年, 研究表明薄 膜磁学及电学性能会受到表面形貌、生长温度、薄 膜厚度、氧空位和祄底应力等因素的影响 ${ }^{[9-12]}$ 。人 们一般选择 $\mathrm{SrTiO}_{3} 、 \mathrm{NdGaO}_{3} 、 \mathrm{SmScO}_{3} 、 \mathrm{KTaO}_{3}{ }^{[13-15]}$ 等单晶氧化物祄底, 生长外延结构的 SRO 薄膜, 重 点研究外延应力对 SRO 薄膜的影响。而对于广泛应 用于半导体集成工艺中的硅 $(\mathrm{Si})$ 衬底上生长的 SRO 薄膜的应力及性能的研究则鲜见报道。本工作采用 射频磁控溅射法, 分别在 $\mathrm{SrTiO}_{3}(\mathrm{STO})$ 单晶衬底和 $\mathrm{Si}$ 祄底上制备了不同取向的 SRO 薄膜, 对其应力状 态进行了分析, 并系统研究了残余应力对 SRO 薄膜 磁学及电输运特性的影响。

\section{1 实验方法}

首先, 采用直流磁控溅射法在二氧化硅/硅 $\left(\mathrm{SiO}_{2} / \mathrm{Si}\right)$ 祄底上制备了一层具有高度 $(001)$ 择优取向 的 $\mathrm{LaNiO}_{3}(\mathrm{LNO})$ 薄膜作为缓冲层来诱导 $\mathrm{SrRuO}_{3}$ 薄 膜的取向生长。接着, 采用射频磁控溅射法分别在 (100) $\mathrm{SrTiO}_{3}$ (STO)、(110) $\mathrm{SrTiO}_{3} 、 \mathrm{SiO}_{2} / \mathrm{Si}$ 和 $\mathrm{LNO} /$ $\mathrm{SiO}_{2} / \mathrm{Si}$ 祄底上制备厚度均为 $240 \mathrm{~nm}$ 的 $\mathrm{SRO}$ 薄膜。 通过传统固相法制备溅射用靶材, SRO 靶材为成分 符合化学计量比的 $\mathrm{SrRuO}_{3}$ 陶瓷。原料为 $\mathrm{SrCO}_{3}$ 和 $\mathrm{RuO}_{x}(\mathrm{Ru} \geqslant 75.0 \%)$ 。靶材烧结条件为 $1500^{\circ} \mathrm{C}$ 保温 $12 \mathrm{~h}$, 升降温速率均为 $1^{\circ} \mathrm{C} / \mathrm{min}$ 。沉积薄膜之前, 首先将祄 底材料进行清洗, 依次在丙酮、酒精等有机溶剂中 超声清洗并用去离子水冲洗, 以除去祄底表面的灰 尘和有机杂质。LNO 和 SRO 薄膜的溅射工艺如表 1 所示。溅射完毕后, 腔体中通入氧气, 关闭祄底加热 装置使之自然冷却至室温。

采用日本理学公司的高分辨 $\mathrm{X}$ 射线衍射仪 (HDXRD, SmartLab)对薄膜的结构进行分析 $(\theta-2 \theta$ 模
表 1 LNO 薄膜和 SRO 薄膜的溅射工艺

Table1 Sputtering parameters of LNO and SRO films

\begin{tabular}{ccc}
\hline Sputtering parameters & LNO & SRO \\
\hline Base pressure $/ \mathrm{Pa}$ & $5 \times 10^{-4}$ & $5 \times 10^{-4}$ \\
Deposition pressure/Pa & 3 & 10 \\
Power $/ \mathrm{W}$ & 80 & 80 \\
$\mathrm{O}_{2} /\left(\mathrm{Ar}+\mathrm{O}_{2}\right)$ & $20 \%$ & $20 \%$ \\
Substrate temperature $/{ }^{\circ} \mathrm{C}$ & 450 & 600 \\
\hline
\end{tabular}

式和 $\varphi$-scan 模式)。采用综合物性测试系统(PPMS-9, Quantum Design, San Diego, CA)测量样品磁化强度和 表面电阻率随温度的变化特性(温度范围为 5 300 K), 其中磁性测量过程中采用的是有场冷却升温测量的 方式(测试磁场方向与样品表面垂直), 表面电阻率 则通过标准四探针法获得。

\section{2 结果与讨论}

\section{1 结构特性}

图 1 所示为不同祄底上制备的 SRO 薄膜的 X 射线 衍射(XRD)图谱,从图中可以看出所有 SRO 薄膜均为纯 钙铁矿相, 并无杂相出现。(100) $\mathrm{SrTiO}_{3}$ 和 $\mathrm{LNO} / \mathrm{SiO}_{2} /$
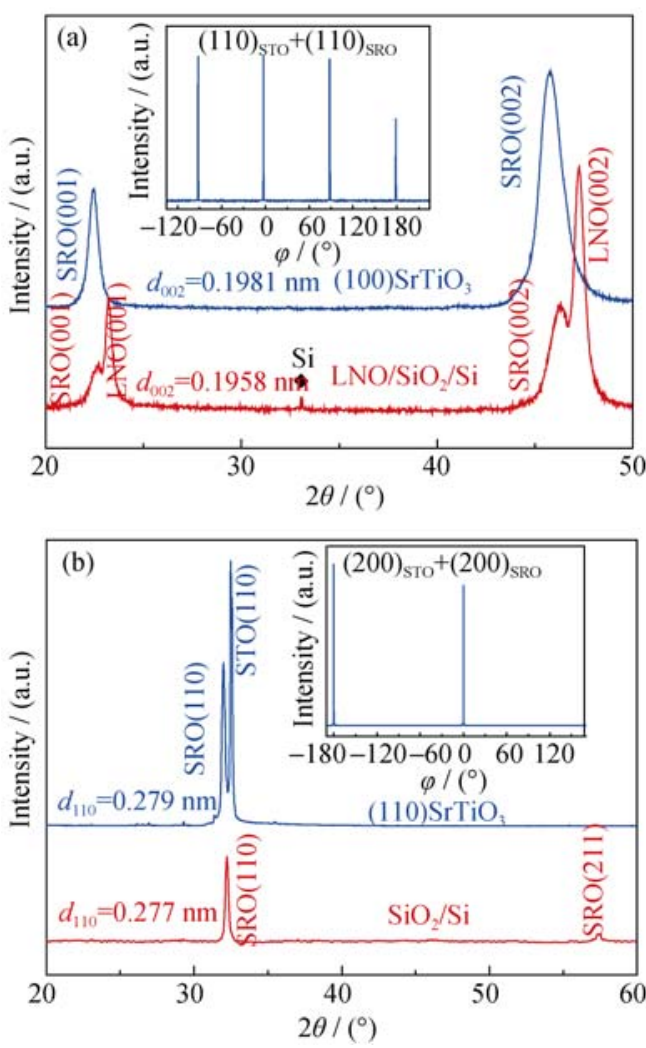

图 1 不同衬底上沉积的 SRO 薄膜的 XRD 图谱

Fig. 1 XRD patterns of $\mathrm{SrRuO}_{3}$ thin films deposited on different substrates

(a) (100) $\mathrm{SrTiO}_{3}$ and $\mathrm{LNO} / \mathrm{SiO}_{2} / \mathrm{Si}$; (b) (110) $\mathrm{SrTiO}_{3}$ and $\mathrm{SiO}_{2} / \mathrm{Si}$ 
$\mathrm{Si}$ 上生长的 SRO 薄膜具有高度的(001)择优取向, 而 (110) $\mathrm{SrTiO}_{3}$ 和 $\mathrm{SiO}_{2} / \mathrm{Si}$ 上生长的 $\mathrm{SRO}$ 薄膜则具有高 度的(110)取向。同时, 还可发现硅基 SRO 薄膜的 衍射峰相对 STO 基 SRO 薄膜发生了明显右移, 说 明薄膜受到不同应力作用。根据 XRD 衍射峰的位 置, 利用布拉格公式可以计算出薄膜的面外晶格 常数。对于硅基(001)SRO 薄膜, 其面外晶格常数 $c=0.3916 \mathrm{~nm}$; 而 STO 基(001)SRO 薄膜的面外晶格 常数 $c=0.3961 \mathrm{~nm}$ 。同样地, 对于(110)取向的 SRO 薄膜, 硅基薄膜的 $<110>$ 面的晶面间距 $d_{110}=0.277 \mathrm{~nm}$, 而 STO 基薄膜的 $<110>$ 面的晶面间距 $d_{110}=0.279 \mathrm{~nm}$ 。 另外, 根据 $\varphi$-Scan 的结果可以确定 STO 基 SRO 薄膜 为外延结构; 而 Si 基 SRO 薄膜, 虽然具有面外方向 上的高度取向, 却由于晶格不匹配, 难以实现外延 生长, 形成的是多晶的单轴取向薄膜。

\section{2 应力分析}

根据由 XRD 结果得到的面外晶格常数值 $c$, \#以 及 SRO 块材的晶格常数 $c_{0}=0.393 \mathrm{~nm}$, 结合面内应 变 $\varepsilon$ 的公式: \#

$$
\varepsilon=\left(c-c_{0}\right) / c_{0} \times 100 \%
$$

计算出不同 SRO 薄膜的应变值, 如表 2 所示。从 表 2 可以看出, STO 基薄膜的 $\varepsilon>0$, 说明薄膜受到压 应力的作用; 而 $\mathrm{Si}$ 基薄膜的 $\varepsilon<0$, 说明薄膜受到拉 应力的作用。并且, Si 基上不同取向的 SRO 薄膜的 $\varepsilon$ 值较为接近, 而 STO 基上不同取向的 SRO 薄膜 的 $\varepsilon$ 值则差距较大，这可能是因为前者是单轴取向 薄膜，面内方向上无择优取向，各向异性较弱; 而后者为外延薄膜，在面内方向上具有较强的各 向异性。

为了进一步分析不同 SRO 薄膜的应力来源, 我 们绘制了如图 2 所示的示意图。一般来说, SRO 薄
表 2 不同 SRO 薄膜的晶格常数和应变值

Table2 Lattice parameters and strain of different SRO films

\begin{tabular}{ccc}
\hline SRO & $c / \mathrm{nm}$ & $\varepsilon / \%$ \\
\hline (001) on STO & 0.3962 & 0.814 \\
$(001)$ on Si & 0.3916 & -0.356 \\
$(110)$ on STO & 0.3945 & 0.383 \\
$(110)$ on Si & 0.3917 & -0.336 \\
\hline
\end{tabular}

膜的应力/应变主要来自于三个方面: (1)由于薄膜内 部缺陷, 如氧空位导致的应力; (2)外延薄膜存在晶 格失配导致的外延应力; (3)由于祄底与薄膜的热膨 胀系数不匹配导致的热应力。具体到实验制备的 SRO 薄膜，由于所有薄膜采用相同的溅射工艺，因 此薄膜的缺陷引起的内应力可不做考虑。对于硅基 SRO 薄膜而言, 因其是多晶单轴取向薄膜, 不是外 延薄膜, 所以外延应力可以忽略, 只考虑热应力对 薄膜的影响。根据相关文献 ${ }^{[16-18]}$, STO 的热膨胀系 数约为 $11 \times 10^{-6} / \mathrm{K}, \mathrm{SiO}_{2} / \mathrm{Si}$ 的热膨胀系数约为 $(2.6 \sim 4) \times 10^{-6} / \mathrm{K}$, 而 SRO 自身的热膨胀系数约为 $10.3 \times 10^{-6} / \mathrm{K}$ 。因此, Si 基 SRO 薄膜只受到热应力作 用。STO 祄底材料的晶格常数为 $0.390 \mathrm{~nm}$, 这与 SRO 块体材料的晶格常数 $(0.393 \mathrm{~nm})$ 非常相近, 其 晶格失配率为 $-0.64 \%$ 。所以对于 STO 基 SRO 薄膜 而言, 除了受到热应力作用外, 由于其是外延薄膜, 还会受到外延压应力的作用。

\section{3 磁学特性}

图 3(a)所示为在外加磁场 $0.5 \mathrm{~T}$ 情况下(磁场方 向垂直于样品表面), SRO 薄膜的磁化强度与温度的 关系，从图中可以发现，无论祄底类型，(001)取向 的薄膜的磁化强度在较宽的温度范围内均大于(110) 取向的薄膜，这说明前者的易磁化方向更接近面外 方向，即所加磁场方向。为了深入了解 SRO 薄膜的

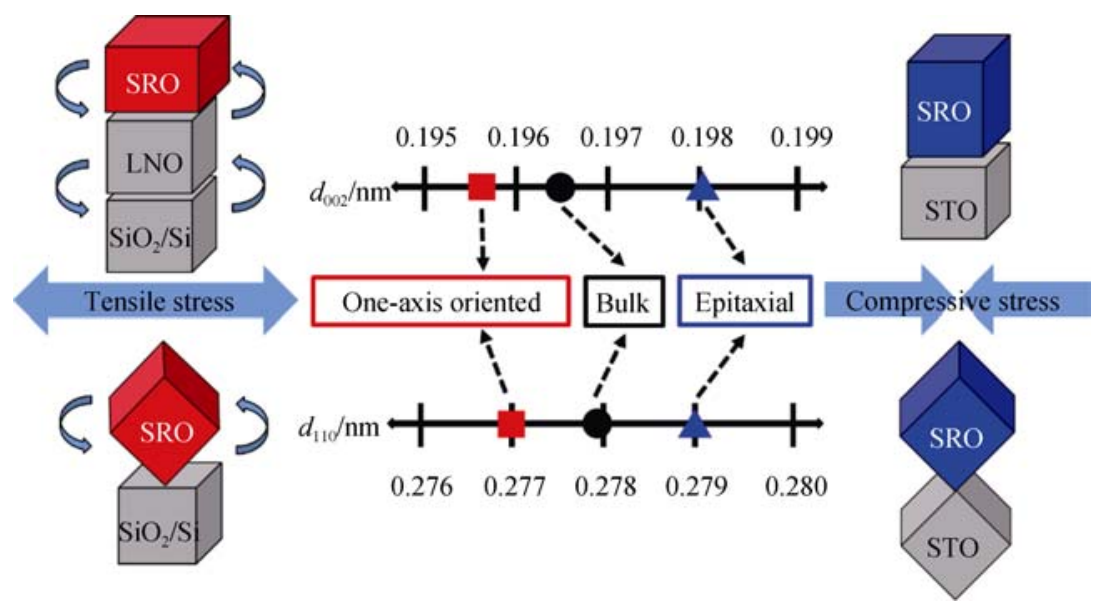

图 2 不同祄底上生长的 SRO 薄膜的应力示意图

Fig. 2 Schematic illustration of stress state for different oriented SRO films on various substates 
磁性能, 对低温下归一化的磁化强度 $M(T) / M(0)$ 随 $T^{3 / 2}$ 的变化关系进行了分析, 如图 2(a)中的插图所 示。可以发现 $M(T) / M(0)$ 与 $T^{3 / 2}$ 呈线性关系, 符合 Bloch 定律 $M(T) / M(0)=1-A T^{3 / 2}$ ( $A$ 为自旋波常数), 这 说明薄膜中自旋波激发占据主导。根据上述得到的 自旋波常数 $A$, 并结合公式: $A=(0.0587 / S)\left(k_{\mathrm{B}} / 2 J S\right)^{3 / 2}$ $\left(S\right.$ 为 $\mathrm{Ru}^{4+}$ 的总自旋数, 这里 $S$ 取 $1^{[19]} ; k_{\mathrm{B}}$ 为玻耳兹曼 常数), 可以计算出表征相邻 $\mathrm{Ru}^{4+}$ 离子交换作用的 $J$ 值。(001)取向的 STO 基和 $\mathrm{Si}$ 基薄膜的 $J$ 值分别为 $11.99 k_{\mathrm{B}} \mathrm{K}$ 和 $10.24 k_{\mathrm{B}} \mathrm{K}$; (110)取向的 $\mathrm{STO}$ 基和 $\mathrm{Si}$ 基 薄膜的 $J$ 值分别为 $8.53 k_{\mathrm{B}} \mathrm{K}$ 和 $9.29 k_{\mathrm{B}} \mathrm{K}$ 。

图 3(b)是磁化强度 $M$ 对温度 $T$ 的求导 $(\mathrm{d} M / \mathrm{d} T)$ 随温度的变化关系图, $\mathrm{d} M / \mathrm{d} T$ 最小值对应的温度即 为该薄膜的居里温度 $\left(T_{\mathrm{C}}\right)$ 。从图中可以看出, $(001)$ 取向的 STO 基薄膜的 $T_{\mathrm{C}}=81 \mathrm{~K}$, 高于(001)的 $\mathrm{Si}$ 基 薄膜的 $T_{\mathrm{C}}=78 \mathrm{~K}$; 但是对于 $(110)$ 取向的薄膜来说, STO 基薄膜的 $T_{\mathrm{C}}=64 \mathrm{~K}$, 低于 $\mathrm{Si}$ 基薄膜的 $T_{\mathrm{C}}=66 \mathrm{~K}$ 。 结合前文应力分析的结果可知, 对于(001)取向的薄 膜, 压应力有助于提高 $T_{\mathrm{C}}$; 而对(110)取向的薄膜而
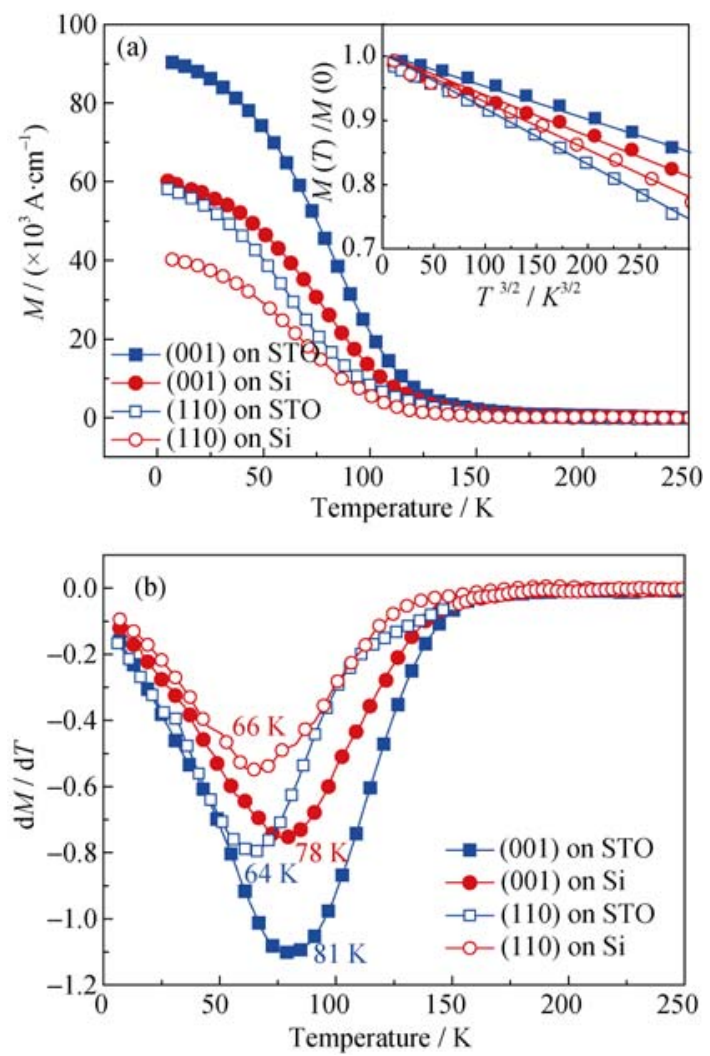

图 3 不同祄底上生长的不同取向的 SRO 薄膜的 (a)磁化强 度随温度的变化曲线和(b)磁化强度对温度的求导值随温度的 变化曲线

Fig. 3 (a) Temperature versus magnetization under field cooling (FC) conditions and (b) the $\mathrm{d} M / \mathrm{d} T$ vs temperature for different oriented SRO films on various substrates
言，压应力却降低了 $T_{\mathrm{C}}$ 。虽然处于相同的应力状态 下，但是由于薄膜取向的差异，不同 SRO 薄膜中 $\mathrm{RuO}_{6}$ 八面体的旋转和倾斜情况不一样, 这就造成 $\mathrm{Ru}-\mathrm{O}$ 键长和键角的差别, 进而导致相邻的 $\mathrm{Ru}^{4+}$ 离 子的交换作用不同，引起了相同应力条件下不同取 向薄膜的 $T_{\mathrm{C}}$ 变化趋势的差异 ${ }^{[20]}$ 。

\section{4 电输运特性}

图 4(a)所示为无外加磁场情况下 SRO 薄膜的归 一化电阻率 $(\rho(T) / \rho(5 \mathrm{~K}))$ 与温度 $(T)$ 的关系。通过归一 化处理, 可以直观地获得不同 SRO 薄膜表面电阻率 的温度依赖性。STO 基(001)和(110)SRO 薄膜的剩 余电阻比 $(R R R=\rho(300 \mathrm{~K}) / \rho(5 \mathrm{~K}))$ 分别为 1.62 和 1.57 ; $\mathrm{Si}$ 基(001)和(110)SRO 薄膜的 $R R R$ 值分别为 1.33 和 1.27。可以看出, 无论 SRO 薄膜的取向如何, STO 基薄膜的表面电阻率均比 $\mathrm{Si}$ 基薄膜呈现出更强的 温度依赖性, 即压应力能够提高电阻的温度依赖性, 而拉应力会弱化这种依赖性，这与 Kan 等 ${ }^{[21]}$ 报道的 结论相一致。同时，对于同一类型祄底上的薄膜而 言, (001)取向的薄膜的 $R R R$ 值均略大于(110)薄膜。 从图 4(a)中还可以发现, 所有 SRO 薄膜均出现了
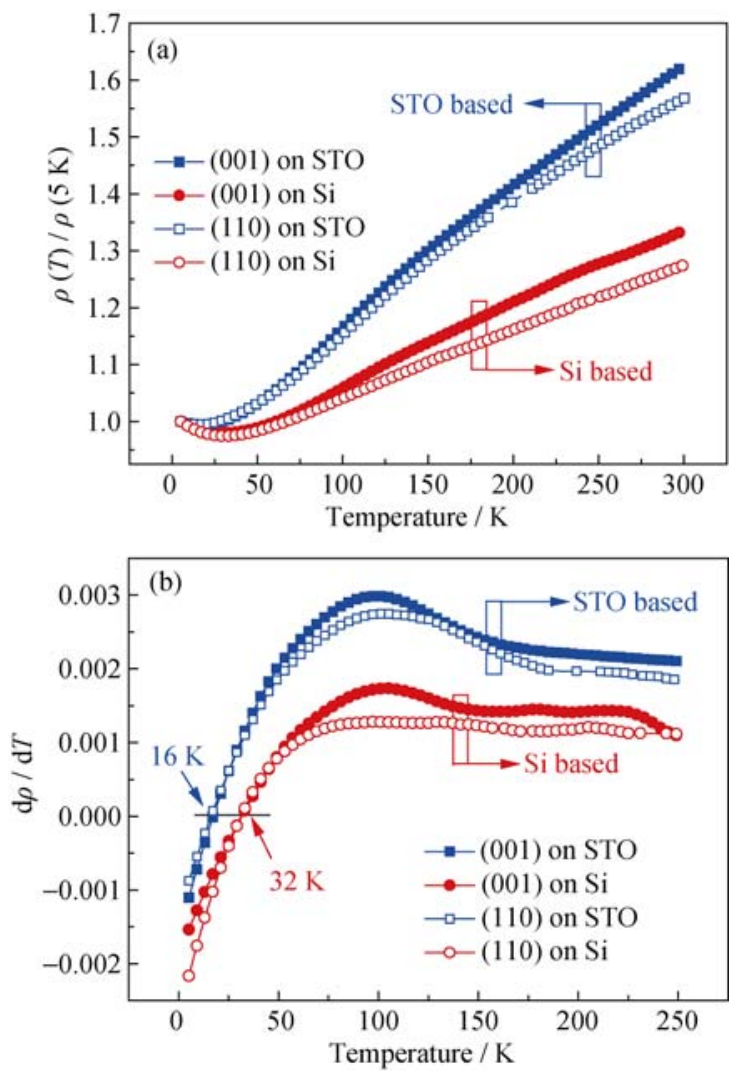

图 4 不同 SRO 薄膜的(a)归一化表面电阻率随温度的变化曲 线和(b)表面电阻对温度的求导随温度的变化曲线

Fig. 4 (a) Temperature versus normalized resistivity and (b) the $\mathrm{d} \rho / \mathrm{dT}$ values $v s$ temperature for different oriented SRO films on various substrates 
“金属-绝缘体相变”。薄膜电阻率随温度的上升而 先下降后上升, 先后呈现出绝缘体和金属导电的 特性。

图 4(b)所示为电阻率对温度的求导 $(\mathrm{d} \rho / \mathrm{d} T)$ 随温 度的变化曲线。 $\mathrm{d} \rho / \mathrm{d} T=0$ 处对应的温度, 即为金属绝 缘体转变温度 $\left(T_{\mathrm{MI}}\right)$ 。由图可知, (001)和(110)取向的 STO 基 SRO 薄膜有着相近的 $T_{\mathrm{MI}}$, 约为 $32 \mathrm{~K}$; 而两 种取向的 $\mathrm{Si}$ 基 $\mathrm{SRO}$ 薄膜的 $T_{\mathrm{MI}}$ 值也较为相近, 约为 $16 \mathrm{~K}$, 低于 STO 基薄膜。根据 Kumar 等 ${ }^{[22]}$ 提出的微 观模型, SRO 薄膜金属-绝缘体转变的出现与薄膜 微结构的失序有关。无序度越大，“局域化效应” 越明显, 进而弱化了表面电阻率对温度的依赖性, 同时 $T_{\mathrm{MI}}$ 也会向高温移动。SRO 薄膜微观结构的无 序度除了与应力有关外, 还与 $\mathrm{STO}$ 基薄膜以及 $\mathrm{Si}$ 基薄膜的晶体结构有关。前者是一种近似于单晶结 构的外延薄膜, 而后者是一种只在面外方向上具有 择优取向的多晶薄膜, 这种结构上的差异导致了不 同祄底上 SRO 薄膜电阻的温度依赖性的差异。

\section{3 结论}

采用磁控溅射法在 STO 祄底和 Si 祄底上制备出 不同取向的 SRO 薄膜, 分析了不同祄底上薄膜的应 力状态, 并进一步研究了剩余应力对薄膜磁学及电 输运特性的影响。

$\mathrm{XRD}$ 测试结果表明, 所有 SRO 薄膜均为典型的 钻钛矿结构, 无杂相。(100) $\mathrm{SrTiO}_{3} 、(110) \mathrm{SrTiO}_{3}$ 祄 底上生长的薄膜分别为(001)和(110)取向的外延薄膜, $\mathrm{LNO} / \mathrm{SiO}_{2} / \mathrm{Si}$ 和 $\mathrm{SiO}_{2} / \mathrm{Si}$ 祄底上生长的薄膜分别为 (001)和(110)取向的多晶单轴取向薄膜;

通过对 SRO 薄膜的应力分析发现, Si 基 SRO 薄 膜受到拉应力作用, 其应力主要为热失配应力, STO 基薄膜受到压应力作用, 其应力除了热失配应力外, 还有外延应力;

通过对其磁学性能的研究表明, (001)取向薄膜 比(110)取向薄膜拥有更高的居里温度 $T_{\mathrm{C}}$; 压应力提 高了(001)取向 SRO 薄膜的 $T_{\mathrm{C}}$, 却降低了 $(110)$ 取向薄 膜的 $T_{\mathrm{C}}$;

对薄膜电输运性能的研究表明, 拉应力引起 了薄膜微结构无序度的增加, 弱化了电阻的温度 依赖性。

\section{参考文献:}

[1] JONES C W, BATTLE P D, LIGHTGOOT P, et al. The structure of $\mathrm{SrRuO}_{3}$ by time-of-flight neutron powder diffraction. Acta
Crystallographica Section C, 1989, 45(3): 365-367.

[2] ALLEN P B, BERGER H, CHAUVET O, et al. Transport properties, thermodynamic properties, and electronic structure of $\mathrm{SrRuO}_{3}$. Phys Rev B Condens Matter, 1996, 53(8): 4393-4398.

[3] CHENG H, LIU Z, YAO K, Rectifying behavior in $\mathrm{La}_{2 / 3} \mathrm{Sr}_{1 / 3} \mathrm{MnO}_{3} / \mathrm{MgO} / \mathrm{SrRuO}_{3}$ magnetic tunnel junctions, Applied Physics Letters, 2011, 98(17): 172107.

[4] EOM C B, VANDOVER R B, PHILLIPS J M, et al. Fabrication and properties of epitaxial ferroelectric heterostructures with $\left(\mathrm{SrRuO}_{3}\right)$ Isotropic Metallic Oxide Electrodes. Applied Physics Letters, 1993, 63(18): 2570-2572.

[5] TANG Z H, TANG M H, LV X S, et al. Enhanced magnetoelectric effect in $\mathrm{La}_{0.67} \mathrm{Sr}_{0.33} \mathrm{MnO}_{3} / \mathrm{PbZr}_{0.52} \mathrm{Ti}_{0.48} \mathrm{O}_{3}$ multiferroic nanocomposite films with a $\mathrm{SrRuO}_{3}$ buffer layer. Journal of Applied Physics, 2013, 113(16): 164106.

[6] SINGAMANENI S R, PUNUGUPATI S, PRATER J T, et al. Ferroelectric and ferromagnetic properties in $\mathrm{BaTiO}_{3}$ thin films on $\mathrm{Si}$ (100). Journal of Applied Physics, 2014, 116(9): 094103.

[7] GRIGERA S, PERRY R, SCHOFIELD A, et al. Magnetic field-tuned quantum criticality in the metallic ruthenate $\mathrm{Sr}_{3} \mathrm{Ru}_{2} \mathrm{O}_{7}$. Science, 2001, 294(5541): 329-332.

[8] HERRANZ G., SANCHEZ F, FONTCUBERTA J, et al. Domain structure of epitaxial $\mathrm{SrRuO}_{3}$ thin films. Physical Review B, 2005, 71(17): 174411.

[9] HERRANZ G, MARTINEZ B, Fontcuberta J, et al. Enhanced electron-electron correlations in nanometric $\mathrm{SrRuO}_{3}$ epitaxial films. Physical Review B, 2003, 67(17): 174423.

[10] SHEN X, QIU X B, SU D, et al. Thickness-dependent metal-insulator transition in epitaxial $\mathrm{SrRuO}_{3}$ ultrathin films. Journal of Applied Physics, 2015, 117(1): 015307.

[11] LU W L, HE K H, SONG W D, et al. Effect of oxygen vacancies on the electronic structure and transport properties of $\mathrm{SrRuO}_{3}$ thin films. Journal of Applied Physics, 2013, 113(17): 17E125.

[12] WANG X W, WANG X, ZHANG Y Q, et al. Magnetic anisotropy and metal-insulator transition in $\mathrm{SrRuO}_{3}$ thin films at different growth temperatures. Journal of Applied Physics, 2010, 107(11): 113925.

[13] PALAi R, HUHTTINEN H, SCOTT J F, et al. Observation of spin-glass-like behavior in $\mathrm{SrRuO}_{3}$ epitaxial thin films. Physical Review B, 2009, 79(10): 104413.

[14] ASO R, KAN D, FUJIYOSHI Y, et al. Strong dependence of oxygen octahedral distortions in $\mathrm{SrRuO}_{3}$ films on types of substrate-induced epitaxial strain. Crystal Growth \& Design, 2014, 14(12): 6478-6485.

[15] LU W L, SONG W D, HE K H, et al. The role of octahedral tilting in the structural phase transition and magnetic anisotropy in $\mathrm{SrRuO}_{3}$ thin film. Journal of Applied Physics, 2013, 113(6): 
063901.

[16] NGUYEN M D, DEKKERS M, HOUWMAN E, et al. Misfit strain dependence of ferroelectric and piezoelectric properties of clamped (001) epitaxial $\mathrm{Pb}\left(\mathrm{Zr}_{0.52}, \mathrm{Ti}_{0.48}\right) \mathrm{O}_{3}$ thin films. Applied Physics Letters, 2011, 99(25): 252904.

[17] XU B, YE Y, Cross L E, Dielectric properties and field-induced phase switching of lead zirconate titanate stannate antiferroelectric thick films on silicon substrates. Journal of Applied Physics, 2000, 87(5): 2507-2515.

[18] YAMANAKA S, MAEKAWA T, MUTA H, et al. Thermophysical properties of $\mathrm{SrHfO}_{3}$ and $\mathrm{SrRuO}_{3}$. Journal of Solid State Chemistry, 2004, 177(10): 3484-3489.
[19] KLEIN L, DODGE J, GEBALLE T, et al. Perpendicular magnetic anisotropy and strong magneto-optic properties of $\mathrm{SrRuO}_{3}$ epitaxial films. Applied physics letters, 1995, 66(18): 2427-2429.

[20] LU W, SONG W, YANG P, et al. Strain engineering of octahedral rotations and physical properties of $\mathrm{SrRuO}_{3}$ films. Sci. Rep., 2015, 5: 10245.

[21] KAN D, ASO R, KURATA H, et al. Epitaxial strain effect in tetragonal $\mathrm{SrRuO}_{3}$ thin films. Journal of Applied Physics, 2013, 113(17): 173912.

[22] KUMAR S, MAJUMDAR P. Singular effect of disorder on electronic transport in strongly coupled electron-phonon systems. Physical Review Letters, 2005, 94(13): 136601. 